\title{
Suppression of the pseudoantisymmetry channel in the conductance of telescoped double-wall nanotubes
}

\author{
Ryo Tamura \\ Faculty of Engineering,Shizuoka University, 3-5-1 Johoku, Hamamatsu 432-8561, Japan \\ Yoko Sawai and Junji Haruyama \\ Aoyama Gakuin University, 5-10-1 Huchinobe, Sagamihara, Kanagawa 229-8558, Japan \\ (Received 16 September 2004; revised manuscript received 24 February 2005; published 7 July 2005)
}

\begin{abstract}
The conductance of telescoped double-wall nanotubes (TDWNTs) composed of two armchair nanotubes $\left[\left(n_{O}, n_{O}\right)\right.$ and $\left(n_{O}-5, n_{O}-5\right)$ with $\left.n_{O} \geqslant 10\right]$ is calculated using the Landauer formula and a tight binding model. The results are in good agreement with the conductance calculated analytically by replacing each single-wall nanotube with a ladder, as expressed by $\left(2 e^{2} / h\right)\left(T_{+}+T_{-}\right)$, where $T_{+}\left(T_{-}\right)$is the transmission rate of the pseudosymmetry (pseudoantisymmetry) channel. Interlayer hopping of the - channel in the ladder model explains two key results: the low value of $T_{-}$for TDWNTs except when $n_{O}=10$, and the particularly low value of $T_{-}$ when either $n_{O}$ or $n_{O}-5$ is a multiple of three. In the latter case, $T_{-}$becomes zero when the saturation of the number of interlayer bonds per atom is neglected.
\end{abstract}

DOI: 10.1103/PhysRevB.72.045413

PACS number(s): 73.63.Fg, 85.35.Kt, 73.22.-f

\section{INTRODUCTION}

The miniaturization of electronic devices will reach real physical limits in the near future, such as the breakdown of ultrathin leads at high current densities. Carbon nanotubes (NTs), with the ability to carry much higher current densities than metal, are therefore expected to become an important component in future devices. This improved current capacity derives from the strong covalent bonds that make up the honeycomb lattice of the NTs. Metallic and semiconducting NTs have also been developed, representing potentially important elements of electronic circuitry. ${ }^{1}$ While the general applicability of single-walled nanotubes (SWNTs) in electronic circuits is feasible, the problem of assembling SWNTs into complex systems remains to be overcome. For example, seamless junctions with disclinations, ${ }^{2,3}$ as well as cross junctions, ${ }^{4}$ and $\mathrm{Y}$-shaped nanotubes ${ }^{5}$ are necessary circuit features. Recently, new types of NT junctions have been prepared from multiwall nanotubes (MWNTs) by electrical breakdown of successive layers ${ }^{6}$ and "telescoping" of the layered MWNT. ${ }^{7,8}$ In such telescoped MWNTs (TMWNTs), the inner core NT is attached to a scanning probe tip and pulled out from the outer NT. In these systems, the SWNT is assembled by interlayer interaction. Thus, the nature of the interlayer interaction is important in terms of both the electronic characteristics and the mechanical properties of the resultant SWNT network. ${ }^{9}$ Double-wall nanotubes (DWNTs) are a special type of MWNT that are prepared from a $\mathrm{C}_{60}$-filled SWNT (Ref. 10) or by catalytic chemical vapor deposition of acetylene with zeolite, ${ }^{11}$ and the energy bands ${ }^{12}$ and conductance ${ }^{13}$ of such DWNTs have been investigated theoretically.

The present paper discusses the electronic characteristics of telescoped double-wall nanotubes (TDWNTs) as the most simple example of a TMWNT (Fig. 1). In a TDWNT, the path of the current along each SWNT is broken between the source electrode and the drain electrode to force the net cur- rent to flow between the layers. Thus, the effect of interlayer connection is much stronger than in untelescoped DWNTs. This enhanced effect is suitable for controlling the current by modifying the interlayer configuration. Furthermore, the interlayer configuration can be controlled directly with angstrom accuracy by adjusting the exact position on the scanning probe to which the TDWNT is attached, as the honeycomb lattice of the TDWNT is resistant to deformation as long as the inner and outer SWNTs are maintained parallel. This stability originates from the stronger intralayer $\sigma$ bond compared to the interlayer connection, making repeatable control of the electronic current possible.

Although the conductance of TDWNTs has been calculated using the Landauer formula, ${ }^{14-17}$ there remains some controversy regarding the result. For example, the maximum conductance of a TDWNT composed of a $(10,10)$ armchair NT and a $(5,5)$ NT is less than $G_{0}$ in Ref. 14 , but close to $2 G_{0}$ in Ref. 16, where $G_{0}$ represents the quantum conductance $2 e^{2} / h$. In other words, the two conduction channels (related to the mirror plane along the tube axis) are open in the former, while one is closed in the latter. Although

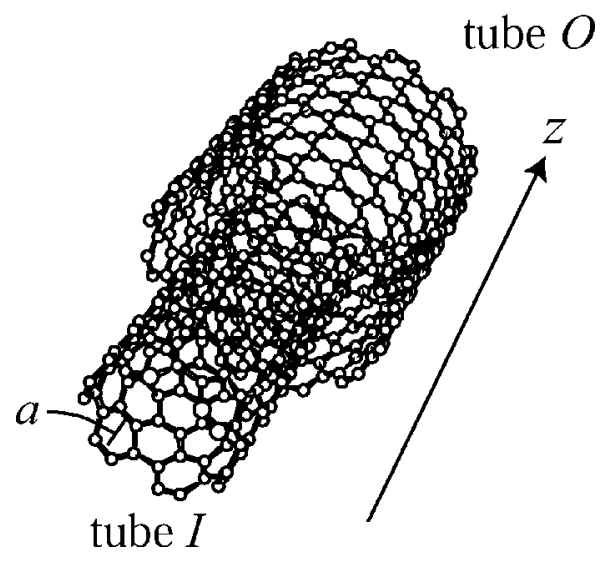

FIG. 1. Telescoped double-wall nanotube. 


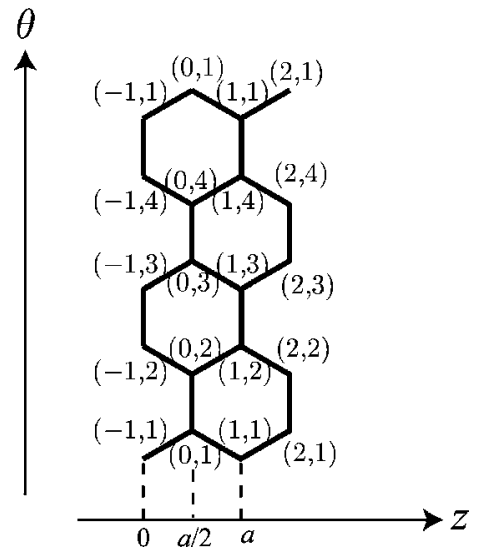

FIG. 2. Index $(l, i)$ representing $z$ and $\theta$ in a $(2,2)$ armchair nanotube.

changes of the interlayer configuration alter the conductance significantly, even when the change is smaller than an angstrom, this behavior was not discussed in either of these studies. ${ }^{14,16}$ For the $(10,10)-(5,5)$ TDWNT, it is shown in this study that both channels remain open in most interlayer configurations, and one channel is closed only when the interlayer configuration satisfies a severe condition. For other TDWNTs, however, the effective channel number is close to one in most cases.

In this paper, the conductance of a TDWNT composed of two armchair nanotubes is calculated using a tube model and a ladder model. The tube model represents the structure of the TDWNT directly and provides a numerical result, whereas the ladder model provides analytical results to derive a physical picture not found in other theoretical papers. ${ }^{14-17}$ Furthermore, in contrast to other theoretical works where a parameter has to be determined by fitting the analytical expression into the numerical results ( $\epsilon$ in Ref. 14 and $\gamma_{\pi-\pi}$ in Ref. 15), all parameters in the present analysis are derived directly from Hamiltonian of the tube model, making it possible to clarify analytically whether the two conduction channels are indeed open or not.

\section{METHODOLOGY}

The outer and inner tubes are denoted by " $O$ " and " $I$." To simplify the discussion, both tubes are defined as armchair tubes, $\left(n_{O}, n_{O}\right)$ and $\left(n_{I}, n_{I}\right)$. As the interlayer distance must be close to that of graphite, only the case of $n_{O}=n_{I}+5$ is considered here. The common tube axis is defined as the $z$ axis in cylindrical coordinates $(r, \theta, z)$. An atom in tube $\mu(\mu$ $=O, I)$ is expressed as $(\mu, l, i)$, where integers $l$ and $i$ increase with $z$ and $\theta$, respectively. For the $(2,2)$ armchair tube, the correspondence between $(\theta, z)$ and $(l, i)$ is shown in Fig. 2 . Tight binding (TB) models and Landauer formula are used to calculate the conductance in both the tube and ladder models, as shown below.

\section{Tube model}

The amplitude of the wave function at $(\mu, l, i)$ is defined as $\psi_{l, i}^{(\mu)}$. In Fig. 3, $\vec{\psi}_{l}^{(\mu)}$ represents the wave function in a half

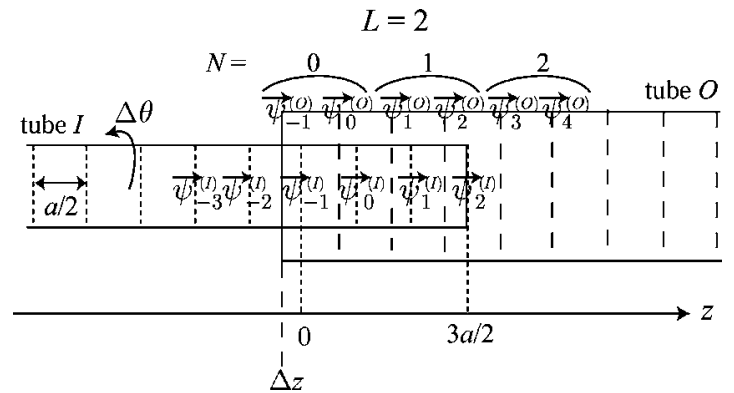

FIG. 3. Schema of a telescoped nanotube and amplitude of the wave functions $\vec{\psi}_{l}^{(O)}$ and $\vec{\psi}_{l}^{(I)}(\Delta z<0)$.

unit cell, as given by ${ }^{t} \vec{\psi}_{l}^{(\mu)}=\left[\psi_{l, 1}^{(\mu)}, \psi_{l, 2}^{(\mu)}, \ldots, \psi_{l, 2 n_{\mu}}^{(\mu)}\right]$. With the lattice constant $a(\simeq 0.25 \mathrm{~nm})$ and a fractional shift $\Delta z(|\Delta z|$ $<0.5 a)$, sites $(I, l, i)$ and $(O, l, i)$ are located at $z=0.5(l$ $+1) a$ and $z=\Delta z+0.5(l+1) a$, respectively. The relative rotation of the two SWNTs is represented by $\Delta \theta \equiv \theta_{1,1}^{(I)}-\theta_{1,1}^{(O)}$, where $\theta_{l, i}^{(\mu)}$ denotes $\theta$ at $(\mu, l, i)$. The maximum value of $l$ for $\vec{\psi}_{l}^{(I)}$ is equal to $2 L-2$, where $L$ represents the number of unit cells in the DWNT region, that is, $L=2$ in Fig. 3. For -1 $\leqslant l \leqslant 2 L-2$, that is, when $l$ is in the DWNT region, " $D$ " is used to express ${ }^{t} \vec{\psi}_{l}^{(D)} \equiv\left[{ }^{t} \vec{\psi}_{l}^{(I)},{ }^{t} \vec{\psi}_{l}^{(O)}\right]$. The wave function in a full unit cell is given by $\vec{e}_{N}$ as follows:

$$
{ }^{t} \vec{e}_{N}=\left[{ }^{t} \vec{\psi}_{2 N}^{(\mu)},{ }^{t} \vec{\psi}_{2 N+1}^{(\mu)}\right]
$$

where $\mu=I$ is the $I$ SWNT region $(N \leqslant-1), \mu=D$ is the central DWNT region $(0 \leqslant N \leqslant L-1)$, and $\mu=O$ is the $O$ SWNT region $(L \leqslant N)$.

The present analysis employs the same TB model with $\pi$ orbitals as used to investigate multilayer graphite and DWNTs in Ref. 18. The intralayer Hamiltonian matrix elements between nearest neighbors are constant values of $-t$ $=-2.75 \mathrm{eV}$, and other intralayer elements are zero. The TB equation for the energy $E$ is expressed as

$$
-P_{2}^{(\mu)} \vec{\psi}_{2 N+2}^{(\mu)}={ }^{t} P_{1}^{(\mu)} \vec{\psi}_{2 N}^{(\mu)}+\left[G_{2}^{(\mu)}-E\right] \vec{\psi}_{2 N+1}^{(\mu)}
$$

and

$$
\left[G_{1}^{(\mu)}-E\right] \vec{\psi}_{2 N+2}^{(\mu)}+P_{1}^{(\mu)} \vec{\psi}_{2 N+3}^{(\mu)}=-{ }^{t} P_{2}^{(\mu)} \vec{\psi}_{2 N+1}^{(\mu)},
$$

under the condition that the Hamiltonian matrix element between $(\mu, l, i)$ and $(\mu, m, j)$ vanishes when $|l-m|>1$. This condition is satisfied when $N \leqslant-2$ and $L \leqslant N$, that is, when $N$ is in the SWNT region. In this case, the matrixes $P_{1}$ and $P_{2}$ become the scalar $-t$. Equations (2) and (3) can then be combined as a matrix equation

$$
A_{\mu} \vec{e}_{N+1}=B_{\mu} \vec{e}_{N}
$$

and the transfer matrix in each SWNT region can be obtained as $T_{\mu}=A_{\mu}^{-1} B_{\mu}$. Solving the eigenvalue problem, $T_{\mu} \vec{u}_{i}^{(\mu)}$ $=\lambda_{i}^{(\mu)} \vec{u}_{i}^{(\mu)}$, the wave function $\vec{e}_{N}$ in the $\mu$ SWNT region can be expressed as 


$$
\vec{e}_{N}=\sum_{i=1}^{2 n_{\mu}}\left[\left(\lambda_{i}^{(\mu)}\right)^{N} \vec{u}_{i}^{(\mu)} x_{i}^{(\mu)}+\left(\lambda_{i+2 n_{\mu}}^{(\mu)}\right)^{N} \vec{u}_{i+2 n_{\mu}}^{(\mu)} y_{i}^{(\mu)}\right],
$$

where $\vec{u}_{i}^{(\mu)}\left[\vec{u}_{i+2 n_{\mu}}^{(\mu)}\right]$ refers to a propagating wave or an evanescent wave moving in the $+z$ direction $(-z$ direction).

As the interlayer interaction is much weaker than the intralayer bond, the interaction is sometimes classified as a van der Waals interaction. Strictly speaking, however, this classification is not correct because there is considerable overlap between the $\pi$ orbitals of neighboring layers, which although irrelevant for van der Waals interaction is essential for allowing interlayer current. That is, without the overlap, the van der Waals force would exist, but the interlayer current would not. Since the overlap gives the interlayer interaction a covalent bond character, the TB model is suitable for representing the electronic state. For example, the density of state of graphite calculated using the TB model for various interlayer configurations has been shown to be consistent with that calculated from first principles. ${ }^{18}$ This covalent character (i.e., anisotropy and a finite number of bonds per atom) can be described by representing the hopping integral between site $i$ in tube $O$ and site $j$ in tube $I$ by

$$
H_{i, j}=W_{i, j} \cos \left(\theta_{i}-\theta_{j}\right) \exp \left[-\left(d_{i, j}-\delta\right) / L_{c}\right]
$$

where $\delta=0.334 \mathrm{~nm}, L_{c}=0.045 \mathrm{~nm}$, and $d_{i, j}$ is the distance between $i$ and $j .{ }^{18}$ The interlayer bonds are classified as AA bonds, $\mathrm{BB}$ bonds, and $\mathrm{AB}$ bonds. For the $\mathrm{AA}$ and $\mathrm{BB}$ bonds, $W_{i, j}=0.36 \mathrm{eV}$, while $W_{i, j}=0.16 \mathrm{eV}$ for the $\mathrm{AB}$ bond. The definitions of these three bond types are as follows. For $d_{i, j}^{2}$ $<d_{0}^{2}+r_{0}^{2}$, where $r_{0}$ is the cutoff distance along the honeycomb lattices $\left(r_{0}=0.36 a / \sqrt{3}\right)$ and $d_{0}$ represents the interlayer distance $5 \sqrt{3} a /(2 \pi)$, an AA bond is formed between $i$ and $j$. This condition guarantees that the number of AA bonds per atom is either zero or one. When an atom has an AA bond, the other interlayer bond of the atom is weakened due to saturation of the covalent bond number. That is, when either site $i$ or site $j$ is connected with the third site $k$ by an AA bond, the bond between site $i$ and site $j$ becomes an AB bond [Figs. 4(c) and 4(d)]. Otherwise, the connection is made by an AA or BB bond [Figs. 4(a) and 4(b)]. In Fig. 4, the atom of the outer tube forms an AA bond when it comes within the area designated by the dashed oval. It should be noted that $H_{i, j}$ in (c) and (d) is smaller than that in (b) for the same $d_{i, j}$ and $\theta_{i}-\theta_{j}$. Hereafter, this character of the interlayer hopping integral is referred to as the "saturation effect," in which $H_{i, j}$ is determined not only by atoms $i$ and $j$ but also by the third atom $k$. The importance of the saturation effect will be discussed later.

There is another cutoff distance $r_{1}$ defined as $1.37 a / \sqrt{3}$ according to Ref. 18 . For $d_{i, j}^{2}>d_{0}^{2}+r_{1}^{2}, W_{i, j}=0$, i.e., the interlayer transfer integral vanishes. As $\Delta z=0$ in the usual stacking of graphite, only the case of small $|\Delta z|$ is considered here. Owing to the cutoff $r_{1}$ and small $|\Delta z|$, it is not necessary to consider the interlayer hopping integral between $\vec{\psi}_{l}^{(O)}$ and $\vec{\psi}_{m}^{(I)}$ when $|l-m|$ is larger than unity. In this case, Eqs. (2)-(4) can also be used for the DWNT region, where 0
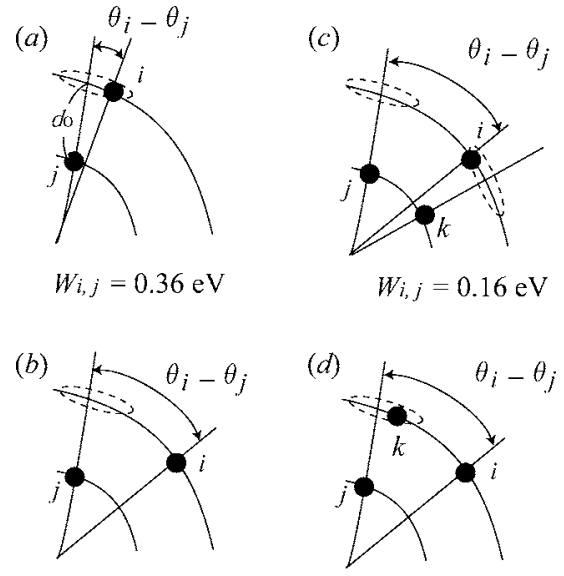

$W_{i, j}=0.36 \mathrm{eV}$

FIG. 4. Saturation effect of the interlayer hopping integral. When the distance between $i$ in tube $O$ and $j$ in tube $I$ is smaller than $d_{0}^{2}+r_{0}^{2}$, the atoms are connected by an AA bond. This threshold is represented by dashed ovals. The other interlayer bonds of an atom with an AA bond are weakened due to saturation of the number of covalent bonds per atom. The hopping integral between $i$ and $j$ in (c) and (d) is smaller than that in (b) for the same sites $i$ and $j$.

$\leqslant N \leqslant L-2$ and $\mu=D$. This gives the transfer matrix in the DWNT region, $T_{D}=A_{D}^{-1} B_{D}$.

Equations similar to Eq. (4) can then be obtained, $A_{I D} \vec{e}_{0}$ $=B_{I D} \vec{e}_{-1}$ and $A_{D O} \vec{e}_{L}=B_{D O} \vec{e}_{L-1}$, at the boundary between the SWNT and DWNT regions. However, the transfer matrix cannot be calculated in this case because $A_{I D}$ and $A_{D O}$ are not square matrices and do not have inverses. In this case, the conditioned transfer matrix can be calculated instead. ${ }^{3}$ The pseudoinverse matrix $\tilde{X}$ is defined as $\tilde{X} \equiv\left(X^{\dagger} X\right)^{-1} X^{\dagger}$ for the matrix $X$ with dimensions $p \times q$ and rank $q(p>q)$. Since $\tilde{X} X=1$ and $\vec{e}_{L-1}=T_{D}^{L-1} \vec{e}_{0}$,

$$
\widetilde{B}_{I D} A_{I D} \vec{e}_{0}=\vec{e}_{-1}
$$

and

$$
\tilde{A}_{D O} B_{D O} T_{D}^{L-1} \vec{e}_{0}=\vec{e}_{L}
$$

However, the vectors $\vec{e}_{0}$ cannot be chosen arbitrary because $X \tilde{X} \neq 1$. The necessary conditions are then

$$
\left(B_{I D} \widetilde{B}_{I D} A_{I D}-A_{I D}\right) \vec{e}_{0}=0
$$

and

$$
\left(A_{D O} \widetilde{A}_{D O} B_{D O}-B_{D O}\right) T_{D}^{L-1} \vec{e}_{0}=0 .
$$

Using Eq. (5) to represent $\vec{e}_{-1}$ and $\vec{e}_{L}$, the wave function of the TDWNT can be expressed by $\vec{x}^{(I, O)}, \vec{y}^{(I, O)}$ and $\vec{e}_{0}$. These vectors have $8\left(n_{I}+n_{O}\right)$ components and must satisfy the conditions (7)-(9), and Eq. (10), where the total number of independent conditions is $6 n_{I}+6 n_{O} ; 4 n_{I}$ in Eq. (7), $4 n_{O}$ in Eq. (8), $2 n_{O}$ in Eq. (9), and $2 n_{I}$ in Eq. (10). Note that not all the rows of the matrix are independent in Eqs. (9) and (10). Using the $4\left(n_{I}+n_{O}\right)$ conditions to eliminate $\vec{e}_{0}$, the other $2\left(n_{I}+n_{O}\right)$ conditions can be represented by 


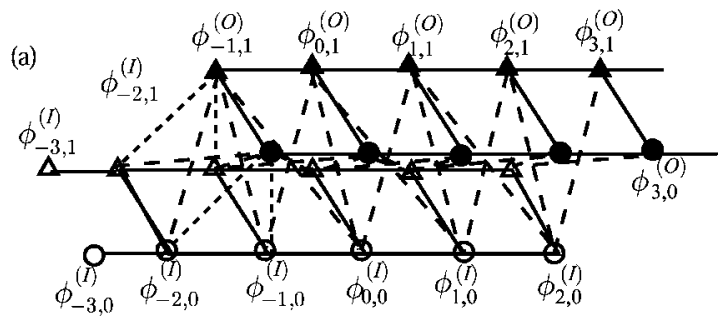

(b)
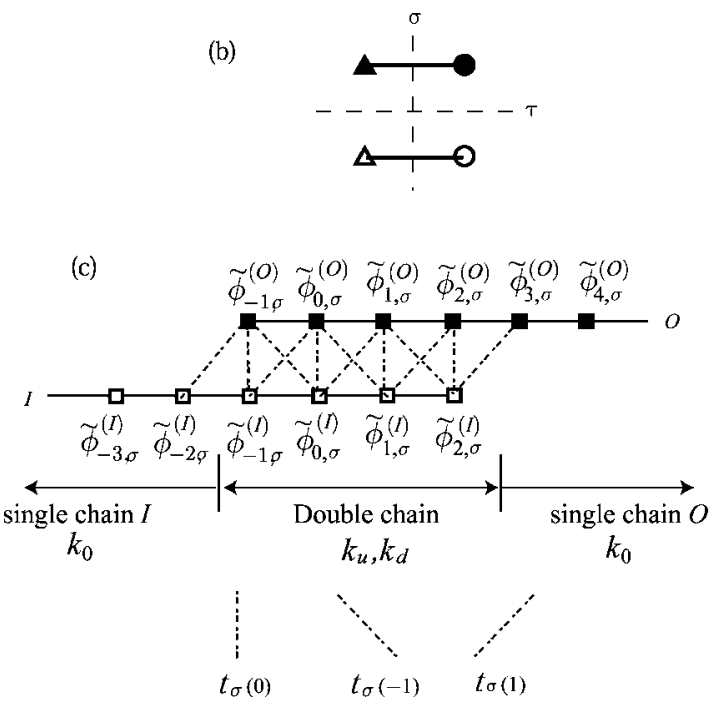

FIG. 5. Schema of ladder model and amplitude of the wave functions $\vec{\phi}_{l}^{(O)}$ and $\vec{\phi}_{m}^{(I)}$. (a) The hopping integrals between the two ladders $h(O, l, i \mid I, m, j)$ are indicated by dashed lines and dotted lines for $i \neq j$ and $i=j$, respectively. For simplicity, the dotted lines are shown only for $l=-1$ and $m=-1,-2$. (b) Mirror planes corresponding to $\sigma=+,-$ and $\tau=u, d$. (c) Ladder model after unitary transformation $\widetilde{\phi}_{l, \sigma}^{(\mu)}=(1 / \sqrt{2})\left(\phi_{l, 0}^{(\mu)}+\sigma \phi_{l, 1}^{(\mu)}\right)$, where $\sigma=+,-$ and $\mu$ $=O, I$.

$$
\left(\begin{array}{l}
\vec{y}^{(I)} \\
\vec{x}^{(O)}
\end{array}\right)=\left(\begin{array}{ll}
S_{I, I} & S_{I, O} \\
S_{O, I} & S_{O, O}
\end{array}\right)\left[\begin{array}{c}
\vec{x}^{(I)} \\
\vec{y}^{(O)}
\end{array}\right]
$$

with the scattering matrix $S$, which describes the outgoing waves $\left[{ }^{t} \vec{y}^{(I)},{ }^{t} \vec{x}^{(O)}\right]$ as a function of the incoming waves $\left[{ }^{t} \vec{x}^{(I)},{ }^{t} \vec{y}^{(O)}\right]$. The cases where $E$ is close to the half-filled Fermi level, that is, $E \simeq 0$, are considered here such that the channel number is two for both the $\left(n_{O}, n_{O}\right)$ and $\left(n_{I}, n_{I}\right)$ NTs. Assigning the propagating waves to the terms with $i=1,2$ in Eq. (5), the conductance is given by $G_{0} \Sigma_{i=1,2} \Sigma_{j=1,2}\left|\left(S_{I, O}\right)_{i, j}\right|^{2}$ according to the Landauer formula with a quantum conductance of $G_{0}=2 e^{2} / h$.

\section{LADDER MODEL}

In the ladder model, tubes $I$ and $O$ are replaced by ladders as shown in Fig. 5. The Hamiltonian of this model $h$ is derived from $H$. Here, $\phi$ is used to represent the wave function in the ladder model to distinguish it from $\psi$ in the tube model. When $i$ and $j$ have common parity, $\phi_{l, i}^{(\mu)}$ corresponds to $\psi_{l, j}^{(\mu)}$. The intralayer elements of $h$ are the same as those in $H$, with nonzero value $-t$ only between the nearest neigh- bors. The interlayer elements of $h$ are defined based on the equation $E \Sigma_{\alpha}\left|\psi_{\alpha}\right|^{2}=\Sigma_{\alpha, \beta} \psi_{\alpha}^{*} H(\alpha \mid \beta) \psi_{\beta}$, which is derived from the TB equation $\left[l, i, \mu\right.$ in $\psi_{l, i}^{(\mu)}$ are abbreviated as $\alpha$ or $\left.\beta\right]$. In comparison with the equivalent equation of the ladder model with the normalization $\left|\vec{\psi}_{l}^{(\mu)}\right|^{2}=\left|\vec{\phi}_{l}^{(\mu)}\right|^{2}$, it can be shown that

$$
\sum_{\alpha, \beta} \phi_{\alpha}^{*} h(\alpha \mid \beta) \phi_{\beta}=\sum_{\alpha, \beta} \psi_{\alpha}^{*} H(\alpha \mid \beta) \psi_{\beta} \text {. }
$$

In Eq. (12), the interlayer terms on the right-hand side are assumed to coincide with those on the left as follows:

$$
h(O, l, i \mid I, m, j)=\sum_{k^{\prime}=1}^{n_{I}} \sum_{k=1}^{n_{O}} \frac{\psi_{l, 2 k+i}^{(O) *} H(\alpha \mid \beta) \psi_{m, 2 k^{\prime}+j}^{(I)}}{\phi_{l, i}^{(O)^{*}} \phi_{m, j}^{(I)}},
$$

where $\alpha=(O, l, 2 k+i)$ and $\beta=\left(I, m, 2 k^{\prime}+j\right)$. Considering plane waves with the normalization $\left|\vec{\psi}_{l}^{(\mu)}\right|^{2}=\left|\vec{\phi}_{l}^{(\mu)}\right|^{2}$, that is, $\left|\psi_{l, i}^{(\mu)} / \phi_{m, j}^{(\mu)}\right|=1 / \sqrt{n_{\mu}}$,

$$
h(O, l, i \mid I, m, j)=\frac{1}{\sqrt{n_{I} n_{O}}} \sum_{k^{\prime}=1}^{n_{I}} \sum_{k=1}^{n_{O}} H^{\prime}(\alpha \mid \beta) .
$$

Here, $\psi_{l, i}^{(\mu)} / \phi_{m, j}^{(\mu)}$ is replaced with $1 / \sqrt{n_{\mu}}$ in order to define $h$ independently of $\phi$ and $\psi$. The Hamiltonian of the tube model $H$ is also replaced by $H^{\prime}$ in the DWNT region, as defined by

$$
H^{\prime}(O, l, i \mid I, m, j)=(1 / 2) \sum_{k=0}^{1} H(O, l-m+k, i \mid I, k, j),
$$

where $-1 \leqslant l \leqslant 2 L-1,-2 \leqslant m \leqslant 2 L-2$ and $l-m=0, \pm 1$. When $l$ and $m$ are outside of this region, $H(O, l, i \mid I, m, j)$ $=H^{\prime}(O, l, i \mid I, m, j)=0$. As a result of this replacement of the Hamiltonian, $h(O, l, i \mid I, m, j)$ with fixed $i$ and $j$ depends only on $l-m$ in the double-ladder region. By unitary transformation, $\widetilde{\phi}_{l, \sigma}^{(\mu)}=(1 / \sqrt{2})\left[\phi_{l, 0}^{(\mu)}+\sigma \phi_{l, 1}^{(\mu)}\right]$ with $\sigma= \pm$, the ladder model can be considered to consist of four chains, $(\mu, \sigma)$, with the following Hamiltonian matrix elements:

$$
\begin{gathered}
\tilde{h}\left(O, l, \sigma \mid I, m, \sigma^{\prime}\right)=\frac{1}{2} \sum_{i=0}^{1} \sum_{j=0}^{1} \sigma^{i}\left(\sigma^{\prime}\right)^{j} h(O, l, i \mid I, m, j), \\
\tilde{h}(\mu, l, \sigma \mid \mu, m, \sigma)=-\sigma t \delta_{l, m}-t\left(\delta_{l, m+1}+\delta_{l, m-1}\right),
\end{gathered}
$$

and $\tilde{h}(\mu, l,-\sigma \mid \mu, m, \sigma)=0$. Due to the simplification represented by Eq. (15), the hopping integral between the chains with common $\sigma$ [i.e., $\tilde{h}(O, l, \sigma \mid I, m, \sigma)]$ depends only on $l$ $-m$ in the double-chain region and is denoted by $t_{\sigma}(l-m)$, as shown in Fig. 5(c). Neglecting small but finite elements between the chains with opposite $\sigma$ [i.e., $\tilde{h}(O, l,-\sigma \mid I, m, \sigma)$ ], the ladder system gains mirror symmetry. As this is approximate symmetry only, the $+(-)$ chain is referred to as a "pseudo" symmetry (pseudoantisymmetry) channel. From this approximation, the analytical transmission rate $T_{\sigma}$ of $\sigma(=+,-)$ channel is given by

$$
T_{\sigma}=\left|\frac{S_{0 u}^{2} \beta_{u}+S_{0 d}^{2} \beta_{d}+C \beta_{u}^{2} \beta_{d}+D \beta_{u} \beta_{d}^{2}}{1-S_{u u}^{2} \beta_{u}^{2}-S_{d d}^{2} \beta_{d}^{2}-2 S_{u d}^{2} \beta_{u} \beta_{d}+\gamma \beta_{u}^{2} \beta_{d}^{2}}\right|^{2},
$$




$$
\begin{gathered}
\beta_{\tau}=\exp \left[i k_{\tau}(2 L-1)\right], \\
C=-\left(S_{0 u} S_{u d}-S_{0 d} S_{u u}\right)^{2}, \\
D=-\left(S_{0 u} S_{d d}-S_{0 d} S_{u d}\right)^{2}, \\
\gamma=\left(S_{u d}^{2}-S_{u u} S_{d d}\right)^{2} .
\end{gathered}
$$

According to the Landauer formula, the conductance is $G_{0}\left(T_{+}+T_{-}\right)$, and the quantum conductance is $G_{0}=2 e^{2} / \mathrm{h}$. Here, $S_{\tau, \tau^{\prime}}$ represents the scattering amplitude from channel $\tau^{\prime}$ to channel $\tau$, where the propagating wave with wave number $k_{\tau}$ corresponds to channel $\tau(\tau=0, u, d)$. Here, $k_{0}$ and $k_{u, d}$ correspond to the plane wave in the single-chain region and the double-chain region, respectively [see Fig. 5(c)]. These values are obtained as a function of the energy $E$ and symmetry $\sigma= \pm$ by solving the following equations:

$$
\begin{gathered}
E=-\sigma t-2 t \cos k_{0}, \\
E=-\sigma t-2 t \cos k_{\tau}+\operatorname{sgn}(\tau)\left|f\left(k_{\tau}\right)\right|,
\end{gathered}
$$

where $\operatorname{sgn}(u)=-\operatorname{sgn}(d)=1$, and $f\left(k_{\tau}\right)(\tau=u, d)$ is the effective interchain hopping defined by

$$
f\left(k_{\tau}\right)=\sum_{j=-1}^{1} t_{\sigma}(j) \exp \left(i k_{\tau} j\right) .
$$

Although $S_{\tau, \tau^{\prime}}$ and $k_{\tau}$ also depend on the symmetry $\sigma=+,-$, this relationship is not shown explicitly in Eq. (17) in order to simplify the notation. The symmetry $\tau=u, d$ originates from the unitary transformation $(1 / \sqrt{2})\left[\alpha_{\tau}^{*} \phi_{l, i}^{(I)}+\alpha_{\tau} \phi_{l, i}^{(O)}\right]$, where

$$
\alpha_{\tau}=\sqrt{\operatorname{sgn}(\tau)} \sqrt{f\left(k_{\tau}\right) * /\left|f\left(k_{\tau}\right)\right|} .
$$

Note that the $\operatorname{sign} \operatorname{sgn}(\tau)$ is independent of the sign $\sigma=+$, -. The mirror plane corresponding to each symmetry is shown in Fig. 5(b). The matrix $S$ is symmetric and unitary, that is, $S^{\dagger}=S^{-1}$ and ${ }^{t} S=S$, and is represented by the phase factors $\alpha_{\tau}$ and $w_{\tau}=\exp \left(i k_{\tau}\right)$ and by the positive group velocity $v_{\tau}=(1 / t)\left(d E / d k_{\tau}\right)>0$ as follows:

$$
\begin{gathered}
S_{0,0}=-\frac{\operatorname{Det}\left[w_{0}^{*}\right]}{\operatorname{Det}}, \\
S_{\tau, \tau}=-\frac{\operatorname{Det}\left[w_{\tau}^{*}, \alpha_{\tau}^{*}\right]}{\operatorname{Det}}, \\
S_{0, \tau}=[-\operatorname{sgn}(\tau) i] \frac{\sqrt{2 v_{0} v_{\tau}}}{\operatorname{Det}}\left(\frac{\alpha_{-\tau}}{w_{-\tau}}\right), \\
S_{u, d}=\sum_{s= \pm} \frac{s}{\operatorname{Det}} \sqrt{\frac{v_{d}}{v_{u}}}\left[\alpha_{u}^{2 s}\left(1-\frac{w_{0}}{w_{u}^{s}}\right)-\frac{t_{\sigma}(1)}{t} w_{0} w_{u}^{s}\right],
\end{gathered}
$$

where
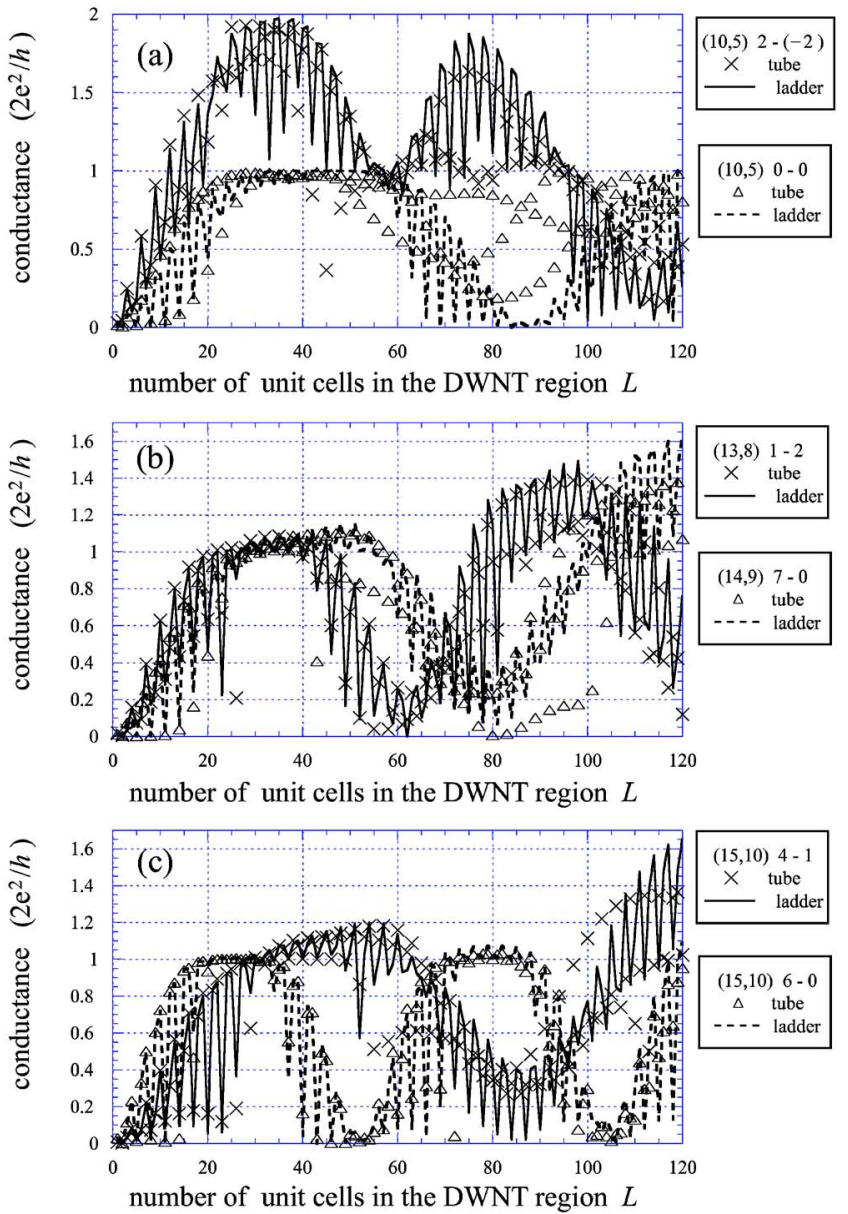

FIG. 6. (Color online) Conductance of the $\left(n_{O}, n_{I}\right) i-j$ TDWNT at $E=0.1 \mathrm{eV}$.

$$
\operatorname{Det}=\sum_{\tau=u, d} \operatorname{sgn}(\tau) \alpha_{-\tau}\left[\frac{1-w_{0} w_{\tau}}{w_{u} w_{d} \alpha_{\tau}}-\frac{t_{\sigma}(1) w_{0}}{t w_{\tau}} \alpha_{\tau}\right]
$$

and $\operatorname{Det}\left[w_{0}^{*}\right]$ in Eq. (26) is defined by Eq. (30) with $w_{0}$ replaced by its complex conjugate $w_{0}^{*}$. The symbols $\operatorname{Det}[\cdots]$ in Eq. (27) have similar meaning.

\section{RESULTS AND DISCUSSION}

Figure 6 shows the conductance of the $\left(n_{O}, n_{I}\right) i-j$ TDWNT for $E=0.1 \mathrm{eV}$ as a function of the number of unit cells of the DWNT $(L)$. Here, $\left(n_{O}, n_{I}\right) i-j$ denotes the TDWNT composed of the tubes $\left(n_{O}, n_{O}\right)$ and $\left(n_{I}, n_{I}\right)$ with rotation angle $\Delta \theta=i 2 \pi /\left(13 n_{O}\right)$ and fractional overlap length $\Delta z$ $=j a /(40)$, where the total overlap length is given by $(L$ $-0.5) a-\Delta z$. There is good agreement between the conductances of the ladder and tube models, verifying that Eq. (17) can be used reliably to discuss the conductance.

Except for the $(10,5)$ TDWNT, the pseudoantisymmetry channel transport is suppressed (the conductance does not reach $4 e^{2} / h$ ) due to the effect of parity cancellation, that is, the terms in Eq. (16) for the pseudoantisymmetry channel $\left(\sigma=\sigma^{\prime}=-1\right)$ with even $i+j$ cancel with those with odd $i+j$ such that the interlayer hopping integral of the pseudoanti- 




(b) $\Delta \theta-0 \quad \diamond-3 \pi / 1000 \quad \times-\pi / 500$

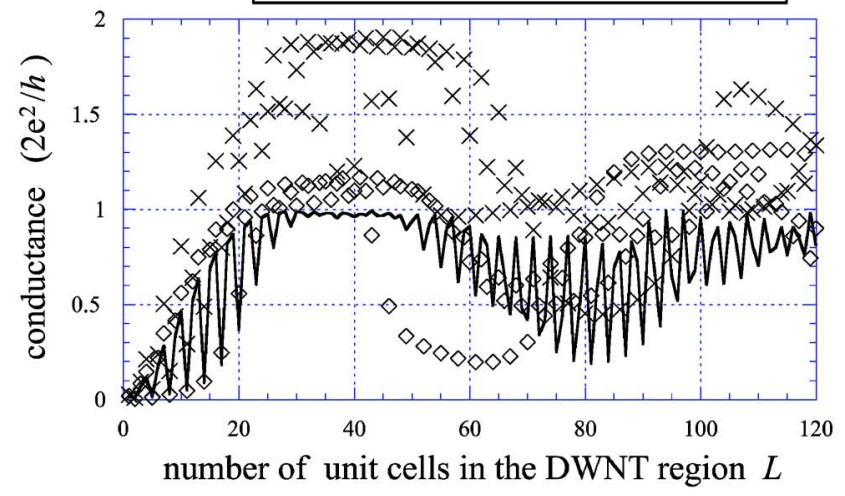

FIG. 7. (Color online) Conductance of the $(10,5)$ TDWNT at $E=0.1 \mathrm{eV}$ calculated using the tube model for $\Delta \theta=-\pi / 500$, $-3 \pi / 1000,0, \pi / 500, \pi / 250$, and $\Delta z=0$. The transmission rate of the pseudoantisymmetry channel is recovered as $|\Delta \theta|$ increases.

symmetry channel $t_{-}$is always smaller than that of pseudo symmetry channel $t_{+}$. This parity cancellation is weakened when $n_{O}\left(=n_{I}+5\right)$ is a multiple of 5 . In order to show this effect, Eq. (16) is rewritten as

$$
\begin{gathered}
t_{-}(l-m)=\sum_{k^{\prime}=1}^{n_{I}} g\left(l-m, k^{\prime}\right), \\
g\left(l-m, k^{\prime}\right) \equiv \frac{1}{\sqrt{n_{l} n_{O}}} \sum_{k=1}^{n_{O}} \sum_{i=0}^{1} \sum_{j=0}^{1}(-1)^{i+j} H^{\prime}(\alpha \mid \beta),
\end{gathered}
$$

where $\alpha=(O, l, 2 k+i)$ and $\beta=\left(I, m, 2 k^{\prime}+j\right)$. When the sign of $g\left(l-m, k^{\prime}\right)$ is changed randomly as a function of $k^{\prime}, t_{-}$ decreases as a result of parity cancellation. When $n_{I}$ is a multiple of five, however, $g\left(l-m, k^{\prime}\right)$ is not random but a periodic function of $k^{\prime}$, as follows:

$$
t_{-}(l-m)=5 \sum_{k^{\prime}=1}^{n_{l} / 5} g\left(l-m, k^{\prime}\right) \text {. }
$$

It is therefore apparent that the parity cancellation is weakened in Eq. (33) compared to the case without fivefold sym-
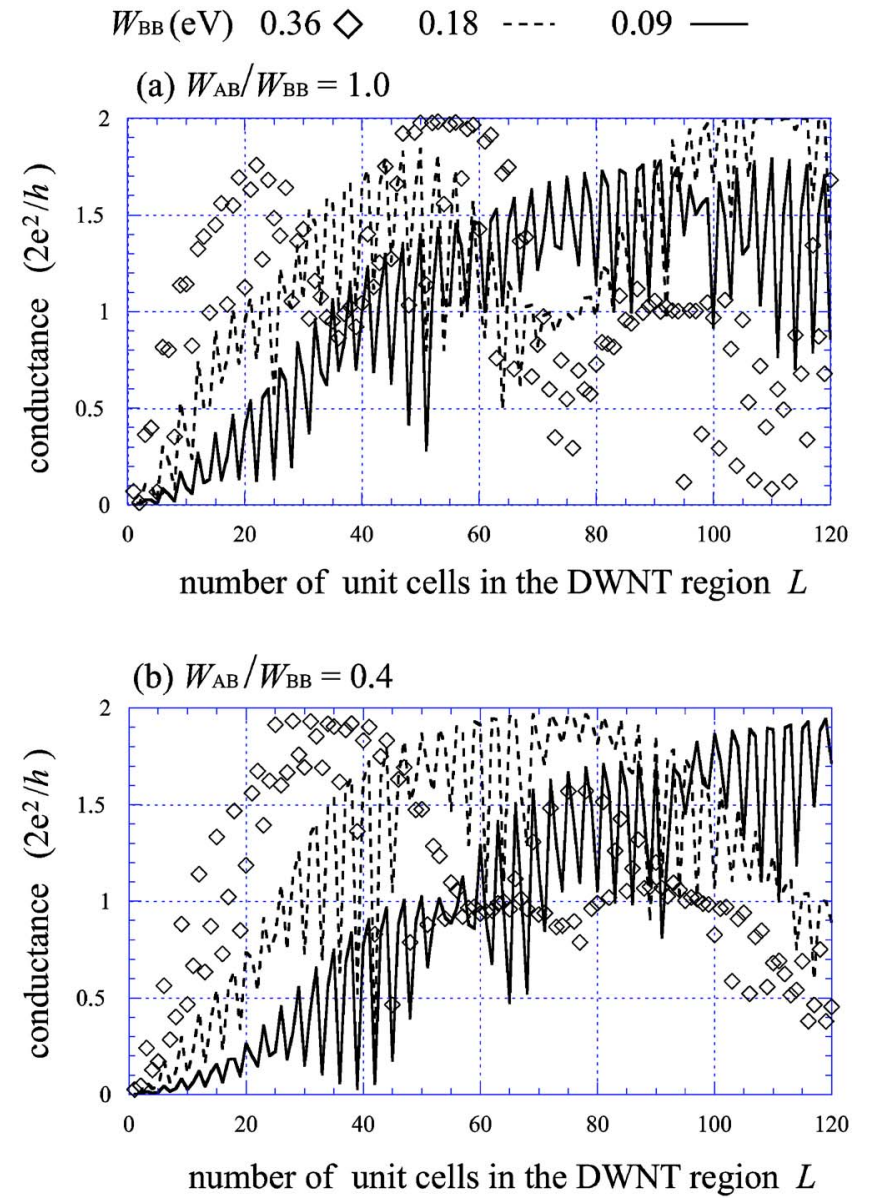

FIG. 8. (Color online) Conductance of the (10,5) 2-(-2) TDWNT at $E=0.1 \mathrm{eV}$ calculated using the tube model for various parameters $W_{\mathrm{BB}}$ and $W_{\mathrm{AB}}$ determining the strength of the interlayer transfer integral in Eq. (6).

metry. This effect is particularly enhanced when $n_{I}=5$, and it is only under this condition that the conductance can reach $4 e^{2} / h$ (Fig. 6).

Even for $n_{I}=5$, however, $T_{-}$is almost zero in the exceptional interlayer configurations $(\Delta \theta, \Delta z) \simeq(0,0),(\pi / 30,0)$ that satisfy

$$
t_{-}(0) \simeq-t_{-}(1) \simeq-t_{-}(-1)
$$

because the effective hopping $f\left(k_{\tau}\right)$ defined by Eq. (24) almost vanishes [see the (10,5) 0-0 TDWNT in Fig. 6(a)]. This can be easily proven by Eqs. (23) and (24), that is, $k_{\tau}$ $\simeq \pi / 3$ and $f\left(k_{\tau}\right) \simeq 0$ satisfy Eqs. (23) and (24) irrespective of $\tau$ under the conditions $E \simeq 0, \sigma=-1$ and (34). Here, "exceptional" means that the area satisfying the condition (34) is very small in the coordination space $(\Delta \theta, \Delta z)$, as shown in Fig. 7. The width of $\Delta \theta$ satisfying the condition (34) is about $\pi / 500$ when $\Delta z=0$. Although Kim and Chang concluded that the maximum conductance of the $(10,5)$ TDWNT is only $G_{0}$, they discussed the exceptional case of $(\Delta \theta, \Delta z)$ $=(\pi / 30,0)$ [Figs. 2(a) and 2(b) in Ref. 14). Their data actually show that the conductance of the $(10,5)$ TDWNT reaches about $1.5 G_{0}$, slightly above the Fermi level [Fig. 2(c) in Ref. 14]. Furthermore, the high conductance of the $(10,5)$ 


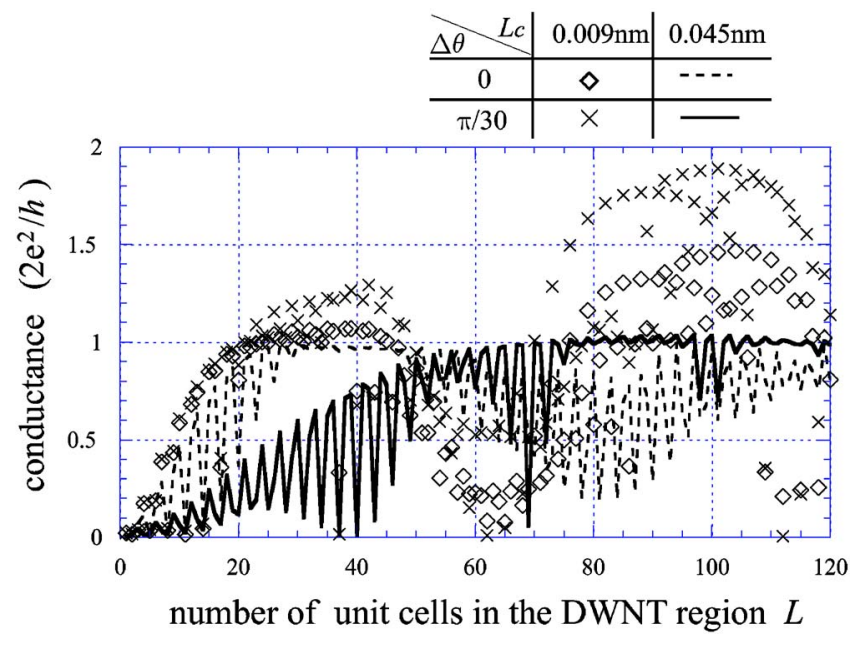

FIG. 9. (Color online) Conductance of the $(10,5)$ TDWNT at $E=0.1 \mathrm{eV}$ calculated using the tube model for the exceptional interlayer configurations $(\Delta \theta, \Delta z)=(0,0),(\pi / 30,0)$. When $L_{c}$ in Eq. (6) is small, the interlayer transfer integral is short range, and the transmission rate of the pseudoantisymmetry channel is large even for these configurations.

TDWNT compared to other TDWNTs, exceeding $G_{0}$ considerably, is not dependent on the details of the TB model employed. For example, the maximum conductance of the $(10,5) 2-(-2)$ TDWNT is close to $2 G_{0}$, irrespective of the strength of the interlayer transfer integrals in Fig. 8. It is also intuitive that the necessary overlap $L$ of the two SWNTs required for the conductance to reach a maximum increases as the interlayer bonds weaken. In contrast to the discussion in Ref. 14, the mirror symmetry is not necessary to realize condition (34). In fact, there is no mirror plane parallel to the axis for $(\Delta \theta, \Delta z)=(0,0)$. The mirror symmetry is also not sufficient, as shown in Fig. 9. That is, even for the configuration with mirror symmetry $(\Delta \theta, \Delta z)=(\pi / 30,0), T_{-}$increases as the interlayer transfer integral becomes shorter ranged. In this case, condition (34) is not satisfied because $\left|t_{-}(1)\right|$ and $\left|t_{-}(-1)\right|$ are reduced compared to $\left|t_{-}(0)\right|$. In Fig. 10 , the dependence of the conductance on the energy is shown for comparison with Fig. 2 of Ref. 14. Figure 10(a) is similar to the graph in Ref. 14, whereas the conductance approaches $2 G_{0}$ with increasing $L$ in Fig. 10(b). The latter case will occur more frequently because the occurrence of $(\Delta \theta, \Delta z)$ satisfying condition (34) is limited.

The parity cancellation is enhanced when either $n_{O}$ or $n_{I}$ is a multiple of 3. This enhanced parity cancellation is referred to as "threefold cancellation." Neglecting the saturation effect for simplicity, $H(O, l, i \mid I, m, j)$ in Eq. (14) with fixed $l$ and $m$ depends only on $\theta_{l_{i}}^{(O)}-\theta_{m, j}^{(I)}$ and can be abbreviated to $H^{\prime}\left[\theta_{l, i}^{(O)}-\theta_{m, j}^{(I)}\right]$, where $\theta_{l, i}^{(O)}$ and $\theta_{m, j}^{(I)}$ represent $\theta$ of sites $(O, l, i)$ and $(I, m, j)$, respectively. Using the notation $\theta_{l, i \pm 2 n_{\mu}}^{(\mu)}=\theta_{l, i}^{(\mu)}$ to represent the periodic boundary condition around the circumference, Eq. (14) can be rewritten as

$$
h(O, l, i \mid I, m, 0)=\frac{1}{\sqrt{n_{I} n_{O}}} \sum_{k^{\prime}=1}^{n_{I}} \sum_{k=1}^{n_{O}} H^{\prime}\left[\theta_{l, 2 k+i}^{(O)}-\theta_{m, 2 k^{\prime}}^{(I)}\right]
$$

and

$$
L=11--\quad L=40 \cdots+110 \quad L
$$

(a) $(10,5) 0-0$
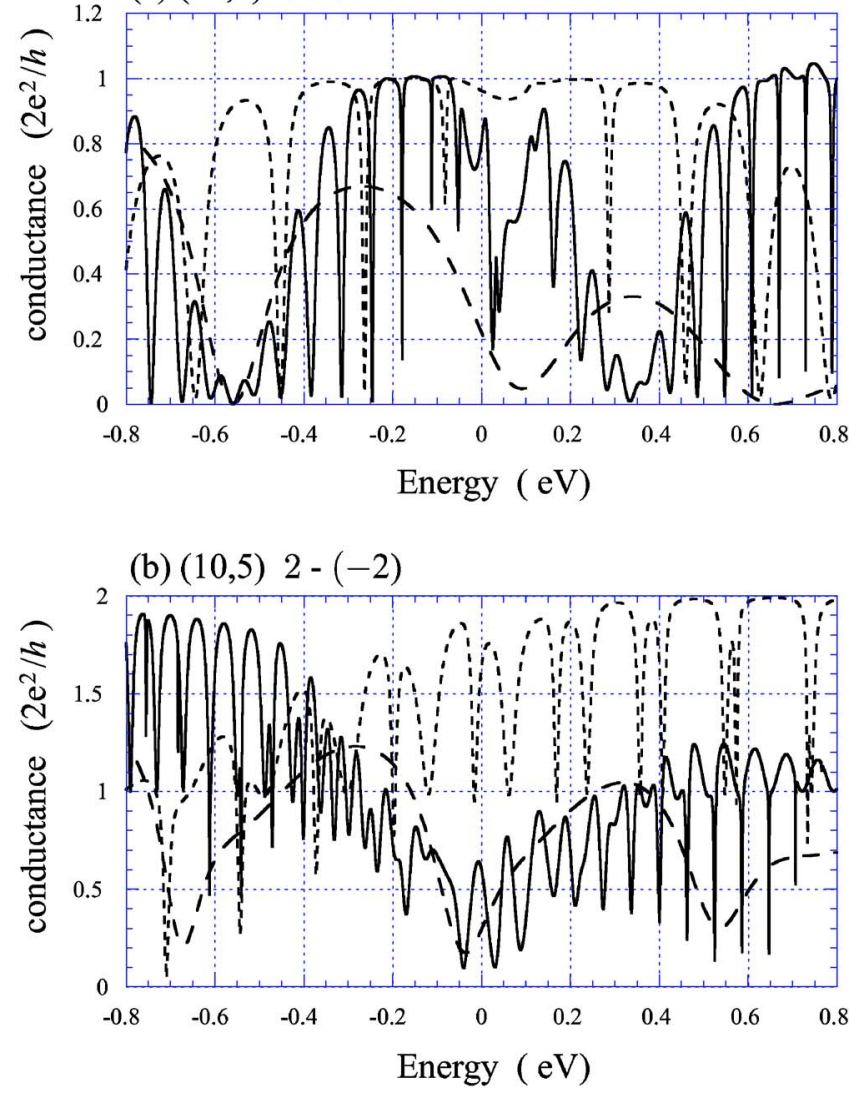

FIG. 10. (Color online) Conductance of (a) the $(10,5)$ 0-0 TDWNT and (b) the $(10,5) 2-(-2)$ TDWNT as a function of energy (calculated using the tube model).

$$
h(O, l, i \mid I, m, 1)=\frac{1}{\sqrt{n_{I} n_{O}}} \sum_{\tilde{k}^{\prime}=1}^{n_{I}} \sum_{\tilde{k}=1}^{n_{O}} H^{\prime}\left[\theta_{l, 2 \tilde{k}+i}^{(O)}-\theta_{m, 2 \tilde{k}^{\prime}+1}^{(I)}\right] .
$$

The following discussion focuses on the case of $n_{O}=n_{I}+5$ as a multiple of 3 . The discussion for the corresponding case of $n_{I}=n_{O}-5$ as a multiple of three can be made in a equivalent manner. From Fig. 2, it can be shown that $\theta_{m, 2 k^{\prime}}^{(I)}-\theta_{m, 2 \tilde{k}^{\prime}+1}^{(I)}$ equals $\left(2 \pi / 3 n_{I}\right)\left(3 k^{\prime}-3 \tilde{k^{\prime}}-2\right)$ for odd $m$ and $\left(2 \pi / 3 n_{I}\right)\left(3 k^{\prime}\right.$ $\left.-3 \tilde{k}^{\prime}-1\right)$ for even $m$. Thus, $\theta_{m, 2 k^{\prime}}^{(I)}-\theta_{m, 2 \tilde{k}^{\prime}+1}^{(I)}= \pm 2 \pi / 3$ when $2 k^{\prime}-\left(2 \tilde{k}^{\prime}+1\right)= \pm\left(2 n_{I}+1\right) / 3$. Here, the upper and lower signs correspond to odd $m$ and even $m$, respectively. On the other hand, $\theta_{l, 2 k+i}^{(O)}-\theta_{l, 2 \tilde{k}+i}^{(O)}= \pm 2 \pi / 3$ when $2 k-2 \tilde{k}= \pm 2 n_{O} / 3$. Relating $k$ and $k^{\prime}$ to $\widetilde{k}$ and $\tilde{k}^{\prime}$ in this way, it can be shown that $\theta_{l, 2 k+i}^{(O)}-\theta_{m, 2 k^{\prime}}^{(I)}=\theta_{l, 2 \tilde{k}+i}^{(O)}-\theta_{m, 2 \tilde{k}^{\prime}-1}^{(I)}$ in Eqs. (35) and (36), that is, $h(O, l, i \mid I, m, 0)$ and $h(O, l, i \mid I, m, 1)$ have the same value and cancel out in $t_{-}(l-m)$ as defined by Eq. (16). This means that the transmission rate $T_{-}$is completely suppressed for arbitrary interlayer configurations $(\Delta \theta, \Delta z)$. This threefold 
(a)

(b)

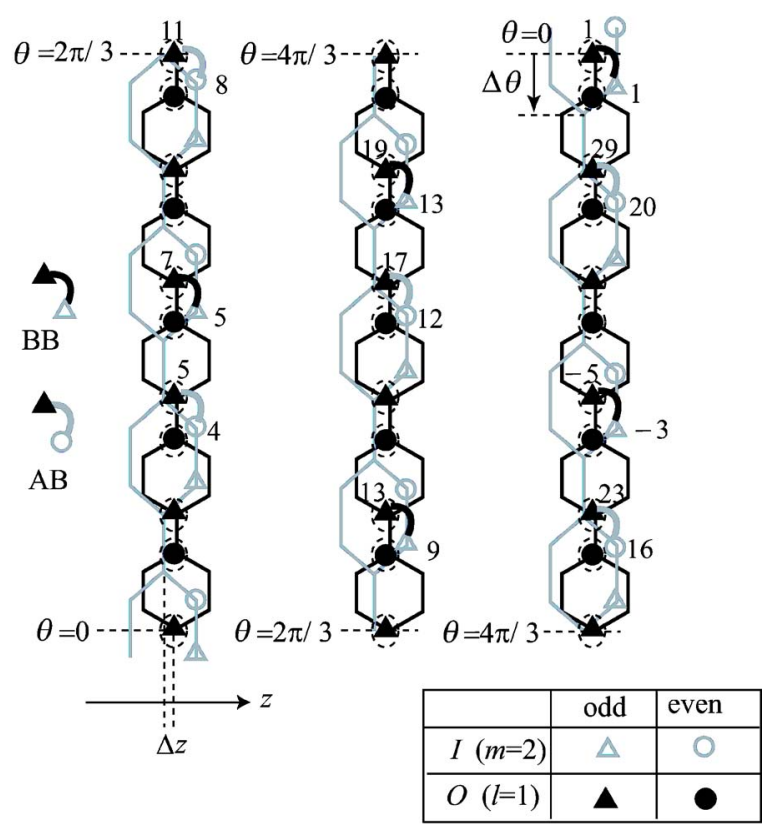

FIG. 11. (Color online) Honeycomb lattice of the $(15,10)$ TDWNT near $z=0$, with $\theta$ as the vertical axis and $z$ as the horizontal axis, for (a) $0 \leqslant \theta \leqslant 2 \pi / 3$, (b) $2 \pi / 3 \leqslant \theta \leqslant 4 \pi / 3$, and (c) $4 \pi / 3 \leqslant \theta$ $\leqslant 2 \pi$. $\Delta z$ is positive and $\Delta \theta=-\pi / 15$. Gray curved lines represent interlayer bonds between $(O, 1,6 p-1)$ and $(I, 2,4 p)$, and black curved lines represent those between $(O, 1,6 p-11)$ and $(I, 2,4 p$ $-7)$, with integers $p=1,2, \ldots 5$. These bonds are labeled with corresponding indices of $\theta(6 p-1,4 p, 6 p-11$, and $4 p-7)$. The curved lines with identical vertical position cancel in the interchain hopping integral of the pseudoantisymmetry channel. Gray and black interlayer bonds denote $\mathrm{AB}$ and $\mathrm{BB}$ bonds, respectively.

cancellation is illustrated in Fig. 11, which shows the honeycomb lattice of the $(15,10)$ TDWNT near $z=0$ with vertical axis $\theta$ and horizontal axis $z$ for (a) $0 \leqslant \theta \leqslant 2 \pi / 3$, (b) $2 \pi / 3$ $\leqslant \theta \leqslant 4 \pi / 3$, and (c) $4 \pi / 3 \leqslant \theta \leqslant 2 \pi$, where $\theta$ and $\theta \pm 2 \pi / 3$ are aligned at the same vertical position. Each pair of interlayer bonds with the same vertical position in Fig. 11 thereby cancels out in $t_{-}(-1)$. For example, substituting $15,1,2$, and 1 for $n_{O}\left(=n_{I}+5\right), l, m$, and $i$, respectively, the hopping integral between $(O, 1,2 k+1)$ and $\left(I, 2,2 k^{\prime}\right)$ cancels that between $(O, 1,2 k-9)$ and $\left(I, 2,2 k^{\prime}-7\right)$ in $t_{-}(-1)$. Figure 11 shows these interlayer bonds for the case of $k=3 p-1$ and $k^{\prime}=2 p$ with integers $p=1,2, \ldots .5$. In this case, the gray and black curved lines correspond to the $(O, 1,6 p-1)-(I, 2,4 p)$ bond and the $(O, 1,6 p-11)-(I, 2,4 p-7)$ bond, respectively. These bonds are labeled with the corresponding index of $\theta$ (i.e., $6 p-1,4 p, 6 p-11$, and $4 p-7$ ). To illustrate the threefold cancellation more clearly, only the vertical positions of the atoms are shown in Fig. 12. For every $2 \pi / 3$ rotation of $\theta$, the vertical position of tube $I$ is shifted by one third of the unit cell, while that of tube $O$ is periodic. This shift aligns each pair of an odd and an even site at a common vertical position in tube $I$.

Taking the saturation effect into account, however, the black curved lines (BB bonds) in Fig. 11 will have a larger hopping integral than the gray curved lines ( $\mathrm{AB}$ bonds),


FIG. 12. Vertical position of $(O, 1, i)$ sites and $(I, 2, j)$ sites in Fig. 11 with respect to $i$ and $j$.

making the threefold cancellation incomplete. In Fig. 11, the classification of the bond is determined by the dashed ovals: when an atom from each of tube $I$ and tube $O$ comes within the dashed oval, an AA bond is formed between them. The dependence of the conductance on $W_{\mathrm{BB}}-W_{\mathrm{AB}}$ is shown in Fig. 13. The saturation effect becomes more pronounced as $W_{\mathrm{BB}}-W_{\mathrm{AB}}$ defined by Eq. (6) increases. For the $(15,10)$ TDWNT, the transmission rate of the pseudoantisymmetry channel $T_{-}$increases with $W_{\mathrm{BB}}-W_{\mathrm{AB}}$, demonstrating that the saturation effect reduces the threefold cancellation. In contrast, Fig. 13(b) shows that the saturation effect does not change the maximum conductance $2 G_{0}$ of the $(10,5)$ TDWNT, as the threefold cancellation does not exist when neither $n_{I}$ nor $n_{O}$ is a multiple of three. This can also be seen by comparing Fig. 8(a) with Fig. 8(b). In this way, the saturation effect influences the maximum conductance only when either $n_{I}$ or $n_{O}$ is a multiple of 3 .

As this discussion suggests, the $(10,5)$ TDWNT has larger maximum conductance due to its fivefold symmetry and lack of threefold cancellation. Although other thicker TDWNTs satisfy the same condition, this effect becomes smaller as $n_{I}$ increases, since the terms $g\left(l-m, k^{\prime}\right)$ in Eq. (33) may be positive or negative according to $k^{\prime}$ and therefore cancel. For example, compare a $(10,5)$ TDWNT with a $(25,20)$ TDWNT, both of which have fivefold symmetry but not threefold cancellation. By calculation using the tube model with $L \leqslant 100$, among the 65 interlayer configurations $(\Delta \theta, \Delta z)=\left[i 2 \pi /\left(13 n_{O}\right), j a / 40\right]$, where $i=0,1, \ldots, 12$ and $j=$ $-2,-1, \ldots 2,31$ configurations have conductance larger than $1.8 G_{0}$ for the $(10,5)$ TDWNT, whereas only 2 exceed this level for the $(25,20)$ TDWNT.

The rapid oscillation and slow variation in the beat structures seen in Fig. 6 originate from the components with large wave numbers $2 k_{u}, 2 k_{d},\left|k_{u}\right|+\left|k_{d}\right|$ and small wave numbers $\left|k_{u}\right|-\left|k_{d}\right|$ in Eq. (17), respectively. As the weak interlayer 
$W_{\mathrm{BB}}=0.36 \mathrm{eV}$

$W_{\mathrm{BB}}-W_{\mathrm{AB}}(\mathrm{eV}) \quad 0 \diamond \quad 0.1 \cdots 0.2-$

(a) $(15,10) 4-1$

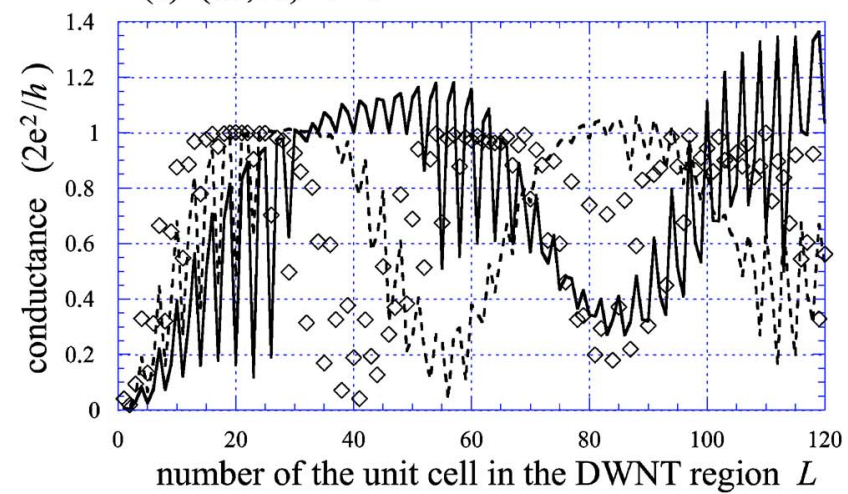

(b) $(10,5) 2-(-2)$

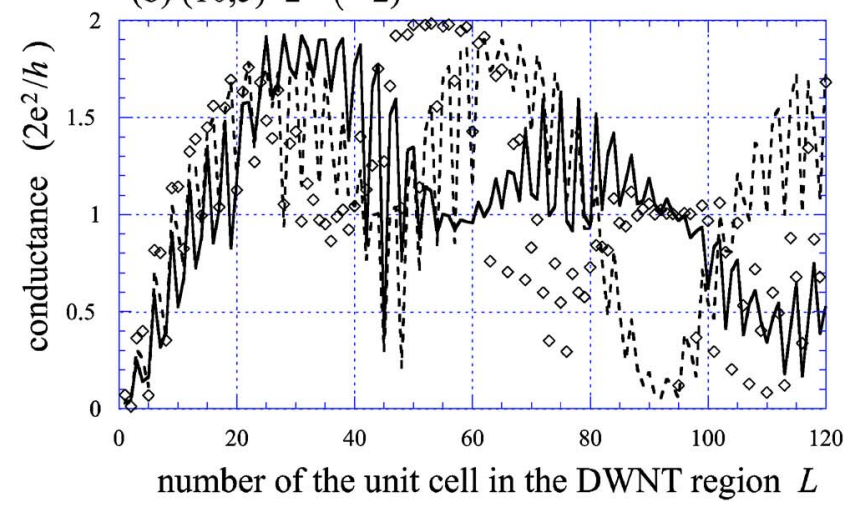

FIG. 13. (Color online) Conductance of the tube model at $E$ $=0.1 \mathrm{eV}$ calculated using the tube model for various values of $W_{\mathrm{BB}}-W_{\mathrm{AB}}$ while $W_{\mathrm{BB}}$ is fixed. The saturation effect becomes more pronounced as $W_{\mathrm{BB}}-W_{\mathrm{AB}}$ increases.

hopping integral modifies the dispersion relation of the NT only slightly, the shift of $\left|k_{u}\right|$ and $\left|k_{d}\right|$ from either $2 \pi / 3$ or $\pi / 3$ is small. Therefore, the period of rapid oscillation is close to three and not much influenced by $\left(n_{O}, n_{I}\right), \Delta \theta$, or $\Delta z$. In contrast, the period of the slow variation varies considerably with these parameters, as even a small change in $k_{u}$ and $k_{d}$ result in large relative changes in $\left|k_{u}\right|-\left|k_{d}\right|$. For example, the phase of the slow oscillation is almost completely reversed by a slight change in $\Delta \theta$ and $\Delta z$ for $40<L<80$ [see Fig. 6(c)]. In such cases, the lattice vibration modulating $\Delta \theta$ and $\Delta z$ might obscure the beat structure, representing a possible reason for the lack of an apparent beat structure in previous experiments. ${ }^{8}$ This sensitivity of the slow variation to $\Delta \theta$ and $\Delta z$ is enhanced by the saturation effect, where the change from a $\mathrm{BB}$ bond to an $\mathrm{AB}$ bond can be induced by a small change in $\Delta \theta$ or $\Delta z$.

Here, it should be noted that the first and second layers of a MWNT can be considered as TDWNTs in series when the first layer is broken down locally. If these two layers are armchair NTs and the current is concentrated in the outermost layer, the pseudoantisymmetry channel of the MWNT is suppressed for the same reason as for the TDWNT. This may be one explanation for the single-channel transmission of MWNTs dipped in liquid metal. ${ }^{19}$ Compared to the explanation considering interlayer interaction given in Ref. 20, the present explanation is relevant for a wider range of Fermi levels.

It is a unique character of TDWNTs that the saturation effect has such a significant influence on the conductance. As the atomic motion changing an $\mathrm{AB}$ bond to a $\mathrm{BB}$ bond cannot be approximated by simple harmonic motion, it can be expected that interlayer vibration will give rise to transport phenomena that differ from the usual phonon-assisted tunneling. Although the effect of interlayer vibration on the conductance in this case is an attractive subject, it will require massive numerical computation. A potential future direction for this work is therefore to extend the ladder model to overcome this difficulty. Intercalated TDWNTs are also attractive, as such a configuration should allow for the detection of current flowing through a small number of molecules or atoms between the layers without the need for an ultrahigh vacuum or low temperatures. A simplified Hamiltonian such as that of the ladder model will also be a powerful tool for this type of analysis.

In conclusion, conductances were calculated for telescoped double-wall nanotubes composed of two armchair nanotubes given by $\left(n_{O}, n_{O}\right)$ and $\left(n_{O}-5, n_{O}-5\right)$ with $n_{O}$ $\geqslant 10$. The interlayer displacement was found to alter the conductance significantly even when the movement is much smaller than the lattice constant. The conductance reaches the maximum value of the two channel system, $4 e^{2} / h$, only when $n_{O}=10$. In other TDWNTs, transport in the pseudoantisymmetry channel is suppressed by parity cancellation. When either $n_{O}$ or $n_{O}-5$ is a multiple of three, $T_{-}$is particularly low with the saturation effect, and exactly zero without the saturation effect. When $n_{O}$ is a multiple of five, the fivefold symmetry reduces the parity cancellation, resulting in an increase in conductance. This effect of fivefold symmetry, however, diminishes with increasing $n_{O}$.
${ }^{1}$ N. Hamada, S. I. Sawada, and A. Oshiyama, Phys. Rev. Lett. 68, 1579 (1992); J. W. Mintmire, B. I. Dunlap, and C. T. White, ibid. 68, 631 (1992); R. Saito, M. Fujita, G. Dresselhaus, and M. S. Dresselhaus, Phys. Rev. B 46, 1804 (1992).

${ }^{2}$ Z. Yao, H. W. C. H. Postma, L. Balents, and C. Dekker, Nature (London) 402, 273 (1999); R. Tamura, Phys. Rev. B 67, 121408(R) (2003); 64, 201404(R) (2001); H. Matsumura and T.
Ando, J. Phys. Soc. Jpn. 67, 3542 (1998); L. Chico, Vincent H. Crespi, Lorin X. Benedict, Steven G. Louie, and Marvin L. Cohen, Phys. Rev. Lett. 76, 971 (1996).

${ }^{3}$ R. Tamura and M. Tsukada, Phys. Rev. B 61, 8548 (2000).

${ }^{4}$ Y.-G. Yoon, M. S. C. Mazzoni, H. J. Choi, J. Ihm, and S. G. Louie, Phys. Rev. Lett. 86, 688 (2001); M. Fuhrer, J. Nygard, L. Shih, M. Foreo, Y.-G. Yoon, M. S. C. Mazzoni, H. J. Choi, J. 
Ihm, S. G. Louie, A. Zettl, and P. L. McEuen, Science 288, 494 (2000).

${ }^{5}$ A. N. Andriotis, M. Menon, D. Srivastava, and L. Chernozatonskii, Phys. Rev. B 65, 165416 (2002); M. Menon and D. Srivastava, Phys. Rev. Lett. 79, 4453 (1997).

${ }^{6}$ J. Cumings, P. G. Collins, and A. Zettl, Science 289, 602 (2000); P. G. Collins, M. Hersam, M. Arnold, R. Martel, and Ph. Avouris, Phys. Rev. Lett. 86, 3128 (2001).

${ }^{7}$ J. Cumings and A. Zettl, Science 289, 602 (2000); Phys. Rev. Lett. 93, 086801 (2004).

${ }^{8}$ S. Akita and Y. Nakayama, Jpn. J. Appl. Phys., Part 1 43, 3796 (2004).

${ }^{9}$ R. Saito, M. Matsumoto, T. Kimura, G. Dresselhaus, and M. S. Dresselhaus, Chem. Phys. Lett. 348, 187 (2001); Y. K. Kwon and D. Tomanek, Phys. Rev. B 58, R16001 (1998); W. Guo, Y. Guo, H. Gao, Q. Zheng, and W. Zhong. Phys. Rev. Lett. 91, 125501 (2003); Q. Zheng, J. Z. Liu, and Q. Jiang, Phys. Rev. B 65, 245409 (2002).

${ }^{10}$ S. Bandow, M. Takizawa, K. Hirahara, M. Yudasaka, and S. Iijima, Chem. Phys. Lett. 337, 48 (2001).

${ }^{11}$ T. Hiraoka, T. Kawakubo, J. Kimura, R. Taniguchi, A. Okamoto,
T. Okazaki, T. Sugai, Y. Ozeki, M. Yoshikawa, and H. Shimohara, Chem. Phys. Lett. 382, 679 (2003).

${ }^{12}$ R. Saito, G. Dresselhaus, and M. S. Dresselhaus, J. Appl. Phys. 73, 494 (1993); Y.-K. Kwon and D. Tománek, Phys. Rev. B 58, R16001 (1998); Y. Miyamoto, S. Saito, and D. Tománek, ibid. 65, 041402 (2001).

${ }^{13}$ S. Uryu, Phys. Rev. B 69, 075402 (2004); D.-H. Kim, H.-S. Sim, and K. J. Chang, ibid. 64, 115409 (2001).

${ }^{14}$ D.-H. Kim and K. J. Chang, Phys. Rev. B 66, 155402 (2002).

${ }^{15}$ I. M. Grace, S. W. Bailey, and C. J. Lambert, Phys. Rev. B 70, 153405 (2004).

${ }^{16}$ C. Buia, A. Buldum, and J. P. Lu, Phys. Rev. B 67, 113409 (2003).

${ }^{17}$ A. Hansson and S. Stafstrom, Phys. Rev. B 67, 075406 (2003).

${ }^{18} \mathrm{Ph}$. Lambin, V. Meunier, and A. Rubio, Phys. Rev. B 62, 5129 (2000); J.-C. Charlier, J.-P. Michenaud, and Ph. Lambin, ibid. 46, 4540 (1992).

${ }^{19}$ S. Frank, P. Poncharal, Z. L. Wang, and W. A. de Heer, Science 280, 1744 (1998).

${ }^{20}$ S. Sanvito, Y. K. Kwon, D. Tomanek, and C. J. Lambert, Phys. Rev. Lett. 84, 1974 (2000). 